\title{
Stereological estimation of particle shape from
}

\section{vertical sections}

\author{
Nick Y. Larsen ${ }^{1,2,3}$, Johanna F. Ziegel ${ }^{1,4}$, Jens R. Nyengaard ${ }^{1,2,3}$ and \\ Eva B. Vedel Jensen ${ }^{1,5}$
}

${ }^{1}$ Centre for Stochastic Geometry and Advanced Bioimaging, Aarhus University

${ }^{2}$ Core Centre for Molecular Morphology, Section for Stereology and Microscopy,

Department of Clinical Medicine, Aarhus University

${ }^{3}$ Sino-Danish Center for Education and Research (SDC)

${ }^{4}$ Institute of Mathematical Statistics and Actuarial Science, University of Bern

${ }^{5}$ Department of Mathematics, Aarhus University

\section{Summary}

In the present paper, we describe a new simple stereological method of estimating volume tensors in $3 \mathrm{D}$ from vertical sections. The volume tensors provide information about particle shape in 3D. In a model-based setting, the method requires that the particle distribution is invariant under rotations around the vertical axis. In a designbased approach, where the vertical section is uniformly rotated around the vertical axis, the method provides information about an index of elongation of the particles in the direction of the vertical axis. The method has been implemented on human brain tissue for the analysis of neurons in layer III of the medial frontal gyrus of

Corresponding author: Nick Yin Larsen: Core Centre for Molecular Morphology, Section Stereology and Microscopy, Aarhus University Hospital, Palle Juul-Jensens Boulevard 99, DK-8000 Aarhus C, Denmark. Tel: +45 7845 5714; email: nylarsen@clin.au.dk. 
Brodmann Area 46. In the actual implementation, the new estimator shows similar precision as an earlier estimator, based on an optical rotator design, but it is a factor 3 faster to collect the measurements for the new estimator. Furthermore, the calculations needed for determining the new estimator are much simpler.

Keywords: Particle processes, rotational invariance, shape, stereology, vertical sections, volume tensors.

\section{Introduction}

Recently, stereological methods of estimating particle shape in 3D have been developed for arbitrarily shaped particles (??). The methods use volume tensors of rank 0, 1 and 2, from which ellipsoidal approximations to the particles can be constructed. Earlier methods provided information about shape of 2D particle sections (????). In particular, 2D analogues of volume tensors were used to describe shape of cell sections (???).

In ? and ?, the volume tensors in 3D are estimated from observations in several optical planes through a sample of particles. The design is called the optical rotator and has earlier been used for estimating particle volume and surface area (?).

As shown in the recent book chapter ?, a much simpler alternative method, which is a generalization of the planar vertical rotator (?), can be constructed. This method uses measurements in a single optical plane, passing through a reference point of each sampled particle. As for the classical local stereological methods, a basic assumption for applying the method is thus that a unique reference point can be associated to each particle.

The purpose of our paper is to present this new and simple method to scientists working in optical light microscopy. In a model-based setting, the method requires that the particle distribution is invariant under rotations around the vertical axis. As a new contribution, we show in this paper that the estimators may also be used 
in a design-based setting where the vertical section is uniformly rotated around the vertical axis. In this design-based approach, we do not need to assume rotational invariance and the method provides information about an index of elongation of the particles in the direction of the vertical axis.

The method has been implemented on human brain tissue for the analysis of neurons in layer III of the medial frontal gyrus of Brodmann Area 46. This area was chosen, since it has been the subject of studies related to schizophrenia and depression (??????). Methods of assessing the precision of the new estimator, based on a bootstrap procedure, are also provided.

The paper is organized as follows. First, we introduce the volume tensors. Then, we discuss inference for particle populations and show how the mean particle volume tensors can be estimated, using the planar vertical rotator design. Finally, volume tensor data collected on neurons from a human brain in layer III of the medial frontal gyrus of Brodmann Area 46 are analyzed. Data, using the planar rotator as well as the optical rotator, are available on the same set of neurons. Finally, we discuss our results and further research questions. Some derivations are deferred to two Appendices.

\section{Volume tensors}

In this section, we introduce the volume tensors in $\mathbb{R}^{3}$ and show how they can be used for obtaining information about size, position, shape and orientation of a spatial particle.

Let $k$ be a non-negative integer. The volume tensor of rank $k$ associated with a particle $X$ (compact subset of $\mathbb{R}^{3}$ ) is given by

$$
T_{k}(X)=\frac{1}{k !} \int_{X} x^{k} \mathrm{~d} x
$$

where $x^{k}$ is the symmetric tensor of rank $k$, determined by $x=\left(x_{1}, x_{2}, x_{3}\right) \in \mathbb{R}^{3}$, and 
the integration is with respect to volume (Lebesgue) measure in $\mathbb{R}^{3}$. Here, $x^{0}=1$ and $x^{1}=x$, while $x^{2}$ is the symmetric $3 \times 3$ matrix with elements $\left(x^{2}\right)_{i, j}=x_{i} x_{j}$, $i, j=1,2,3$. For general $k$, the tensor $x^{k}$ can be represented as a $k$-dimensional array. The integration in (??) is to be understood elementwise.

We will focus on volume tensors of rank 0,1 and 2 . The volume tensor of rank 0

$$
T_{0}(X)=\int_{X} 1 \mathrm{~d} x=V(X)
$$

is simply the volume of $X$, while the volume tensor of rank 1 is the following point in $\mathbb{R}^{3}$

$$
T_{1}(X)=\left(\int_{X} x_{1} \mathrm{~d} x, \int_{X} x_{2} \mathrm{~d} x, \int_{X} x_{3} \mathrm{~d} x\right)
$$

It follows that $T_{1}(X) / T_{0}(X)$ is the centre of mass $c(X)$ of $X$, indicating the position of $X$ in $\mathbb{R}^{3}$. The volume tensor of rank 2 can be represented as a $3 \times 3$ matrix with $(i, j)^{\prime}$ th entry

$$
T_{2}(X)_{i, j}=\frac{1}{2} \int_{X} x_{i} x_{j} \mathrm{~d} x, \quad i, j=1,2,3
$$

Combining $T_{0}(X), T_{1}(X)$ and $T_{2}(X)$, we can obtain information about the shape and orientation of $X$. Thus, these tensors can be used to construct a centred ellipsoid $e(X)$ of the same volume as $X$ such that $c(X)+e(X)$ is an ellipsoidal approximation to $X$, cf. Figure ??. If $X$ is an ellipsoid, then $X=c(X)+e(X)$. The ellipsoid $e(X)$ can be determined from a spectral decomposition of $T_{2}(X-c(X))$,

$$
T_{2}(X-c(X))=T_{2}(X)-\frac{T_{1}(X)^{2}}{2 T_{0}(X)}=B \Lambda B^{T},
$$

where $B$ is an orthogonal matrix and $\Lambda$ is a diagonal matrix with diagonal elements $\lambda_{i}, i=1,2,3$. The ellipsoid $e(X)$ is determined by having directions of semi-axes equal to the columns of $B$, lengths of semi-axes proportional to $\sqrt{\lambda_{i}}, i=1,2,3$, and volume equal to $V(X)$. 
— Figure ?? here -

\section{Inference for particle populations}

In the present paper, we are interested in making inference for a particle population at the population level. Parameters of interest are, for instance, mean particle volume and mean particle shape.

We will assume that we can associate a reference point $x(X) \in X$ to each particle $X$. We let $\bar{T}_{k}, k=0,1,2$, be the mean particle volume tensor of rank $k$, where each particle $X$ enters in the mean with its own reference point $x(X)$ as origin. For $k=0$, we get the mean particle volume $\bar{v}=\bar{T}_{0}$, while $\bar{c}=\bar{T}_{1} / \bar{T}_{0}$ is the so-called displacement vector (?, p. 232), containing information about the average difference between the centre of mass and the reference point of the particles in the population. See Figure ?? for an illustration.

Furthermore, a centred ellipsoid $\bar{e}$ can be constructed that provides information about average particle shape and orientation. The ellipsoid $\bar{e}$ is called the Miles ellipsoid after Roger Miles who was a pioneer in the development of stereological methods for particle populations with arbitrarily shaped particles. The Miles ellipsoid is determined from $\bar{T}_{0}, \bar{T}_{1}$ and $\bar{T}_{2}$, using exactly the same method as the one used for determining $e(X)$ from $T_{0}(X), T_{1}(X)$ and $T_{2}(X)$. If the particles are translations of the same particle $X_{0}$, then the Miles ellipsoid is simply the approximating ellipsoid $e\left(X_{0}\right)$. The concept of the Miles ellipsoid is also illustrated in Figure ??. Further illustrations of the displacement vector and the Miles ellipsoid may be found in ?, Fig. 3 .

— Figure ?? here - 
The estimates of the mean particle volume tensors will be based on a random sample of particles. One possibility is to sample all particles with reference point in a 3D sampling window $W$. In the case of disector sampling (?), $W$ may be a set of systematically placed sampling boxes. If we let $S$ be the set of sampled particles, an estimator of $\bar{T}_{k}$ is the following

$$
\frac{1}{N(W)} \sum_{X \in S} T_{k}(X-x(X)),
$$

$k=0,1,2$, where $N(W)$ is the number of sampled particles. The estimator (??) is ratio-unbiased if $W$ has a uniform random position (design-based approach) or the particles can be modelled by a stationary point process model (model-based approach, see Appendix A).

However, for the determination of the estimator (??), we need to be able to determine the volume tensors $T_{k}$ directly on the sampled spatial particles. For the case where we do not have direct access to the particles in $3 \mathrm{D}$, an estimator of $T_{k}$ based on observation in an optical rotator, consisting of several optical planes, has been developed in ?. As we shall see in the next section, a much simpler alternative method can be constructed based on observations in a planar vertical rotator.

\section{Estimation using the planar vertical rotator}

In this section, we present an estimator of $\bar{T}_{k}$ that only uses measurements in vertical planes passing through the reference points of the sampled particles.

The estimator is valid in a model-based setting if the particles can be modelled by a stationary marked point process, satisfying the assumption of rotational invariance with respect to a predetermined fixed axis, called the vertical axis. (We use this terminology for the axis also in cases where it is not vertical.) For the use of the estimator in a design-based set-up, see the next section.

The particle process is rotation invariant, if the particle distribution is invariant 
under rotations around the vertical axis. Under rotational invariance, the vertical axis represents the average orientation of the particles in 3D and, as we shall see, the mean particle shape in 3D can be estimated from observations in vertical planes. The point process model is described in detail in Appendix A where also rotational invariance is formally defined.

The design used for each sampled particle $X$ is a new, innovative application of the planar vertical rotator design (?), involving registration of $3 \mathrm{D}$ coordinates of intersection points. The design consists of a plane, passing through the reference point of the particle, taken here to be the origin $O$. The plane contains the vertical axis, see Figure ??. The section is subsampled by a systematic set of alternating half lines, perpendicular to the vertical axis.

\section{— Figure ?? here -}

In ?, p. 427-429, ratio-unbiased estimators of $\bar{T}_{k}$ are derived under the rotational invariance assumption. (In ?, 'rotational invariance' was called 'restricted isotropy'.) The estimators $\widehat{T}_{k}$ are of the following form

$$
\widehat{T}_{k}=\frac{1}{N(W)} \sum_{X \in S} \widetilde{T}_{k}([X-x(X)] \cap L),
$$

where $L$ is the notation used for the vertical plane. The ratio-unbiasedness relies on the fact that under rotational invariance the distribution of size, orientation and shape of the section profiles $\{[X-x(X)] \cap L\}$ does not depend on the rotation of $L$ around the vertical axis. An illustration of this property may be found in ?, Fig. 10.

In Appendix A, the explicit form of $\widetilde{T}_{k}$ is derived for $k=0,1,2$. Here, we explain the measurements and calculations needed for determining $\widetilde{T}_{k}$. For a sampled particle $X$ with reference point $O, \widetilde{T}_{k}(X \cap L)$ is a sum over half lines. We use the same type of notation as for rotator measurements (?). We number the intersection points between the $i$ 'th half line and the boundary of $X$ according to decreasing distance 
to the vertical axis, using number 0 for the most distant intersection point. We let $l_{i j}$ be the distance from the $j^{\prime}$ th intersection point on the $i$ 'th half line to the vertical axis, cf. Figure ??.

\section{— Figure ?? here -}

Define the so-called squared ray distance for the $i$ 'th half line by

$$
l_{i}^{2}=\sum_{j \text { even }} l_{i j}^{2}-\sum_{j \text { odd }} l_{i j}^{2}
$$

Likewise, the power- 4 ray distance is defined as

$$
l_{i}^{4}=\sum_{j \text { even }} l_{i j}^{4}-\sum_{j \text { odd }} l_{i j}^{4}
$$

Let $z_{i}$ be the (signed) distance from $O$ to the $i$ 'th half line. If we let $t$ be the distance between neighbour half lines and choose a coordinate system such that the vertical plane $L$ is the $x z$-plane and the vertical axis is the $z$-axis, then we have

$$
\begin{aligned}
& \widetilde{T}_{0}(X \cap L)=\pi t \sum_{i} l_{i}^{2}, \\
& \widetilde{T}_{1}(X \cap L)=\left(0,0, \pi t \sum_{i} z_{i} l_{i}^{2}\right), \\
& \widetilde{T}_{2}(X \cap L)=\left(\begin{array}{ccc}
\frac{\pi}{8} t \sum_{i} l_{i}^{4} & 0 & 0 \\
0 & \frac{\pi}{8} t \sum_{i} l_{i}^{4} & 0 \\
0 & 0 & \frac{\pi}{2} t \sum_{i} z_{i}^{2} l_{i}^{2}
\end{array}\right) .
\end{aligned}
$$

In Table ??, the calculations are illustrated for the profile shown in Figure ??.

\section{— Table ?? here -}

The estimator of volume already appeared in ?. Note that the calculations needed 
for determining (??)-(??) are much simpler than the ones appearing in ?, p. 231 where the optical rotator was used instead of the planar vertical rotator.

Under the assumption of rotational invariance, the mean particle volume is estimated by $\widehat{v}=\widehat{T}_{0}$ and the displacement vector by $\widehat{c}=\widehat{T}_{1} / \widehat{T}_{0}$. An estimator $\widehat{e}$ of the Miles ellipsoid can be calculated from $\widehat{T}_{0}, \widehat{T}_{1}$ and $\widehat{T}_{2}$, using the same procedure as the one used for constructing $e(X)$ from $T_{0}(X), T_{1}(X)$ and $T_{2}(X)$.

Note that the estimated displacement vector $\widehat{c}$ is parallel to the vertical axis. Furthermore, the estimated Miles ellipsoid $\widehat{e}$ is an ellipsoid of revolution around the vertical axis, since

$$
\widehat{T}_{2}-\frac{\left(\widehat{T}_{1}\right)^{2}}{2 \widehat{T}_{0}}
$$

is a diagonal matrix with first and second diagonal elements equal. Under rotational invariance, the same is true for the theoretical quantities $\bar{c}$ and $\bar{e}$, see Appendix A.

\section{Relaxing the rotational invariance assumption}

When rotational invariance is satisfied, it is not needed to rotate the vertical planes around the vertical axis. However, if rotational invariance is not a plausible model assumption, one may instead adopt a design-based approach and use vertical planes that are uniformly rotated around the vertical axis. More specifically, rotational invariance may be introduced into the model by letting the vertical plane $L$, used in the estimators $\widehat{T}_{k}$ in (??), have a uniform rotation around the vertical axis. An equivalent description of the situation is that each centred particle $X-x(X)$ is given a random rotation and then sectioned by a fixed vertical plane $L_{0}$, say. The induced particle model satisfies the rotational invariance assumption.

Thus, in a design-based approach, we can use exactly the same measurements and calculations as in the model-based approach. The resulting estimates $\widehat{v}, \widehat{c}$ and $\widehat{e}$, determined as explained in the previous section, now refer to the induced particle population where each centred particle is given a uniform rotation around the 
vertical axis.

Since a rotation does not change volume, $\widehat{v}$ is still an unbiased estimator of the mean particle volume $\bar{v}$. But, unless the original particle population satisfies the rotational invariance assumption, the displacement vector and the Miles ellipsoid in the induced model may differ from those of the original particle model. As explained in Appendix A, the displacement vector in the induced model is equal to the projection onto the vertical axis of the displacement vector $\bar{c}$ in the original particle model, so $\widehat{c}$ becomes an estimator of the projection onto the vertical axis of the displacement vector $\bar{c}$.

In this design-based approach, the estimator $\widehat{e}$ of the Miles ellipsoid in the induced model may be used to estimate an index $I$ of elongation of the particles in the direction of the vertical axis. The Miles ellipsoid in the induced model is an ellipsoid of revolution around the vertical axis. If the lengths of the semi-axes of this ellipsoid, parallel and perpendicular to the vertical axis, are denoted $a$ and $b$, respectively, then the elongation index $I$ is

$$
I=a / b
$$

Large values of $I$ indicate elongation in the direction of the vertical axis. The index $I$ takes the value 1, if the original particle population is isotropic. More details about this index may be found in Appendix A.

\section{Practical implementation of tensors in optical light microscopy}

In this section and the next, we exemplify the estimation of volume tensors, using the design-based approach with the planar vertical rotator, as explained in the previous sections. The resulting estimator (??) of the volume tensor of rank $k$ will here be called the section estimator. We will compare the performance of the section 
estimator with that of the estimator developed in ?, based on the optical rotator design. The latter estimator will be called the slice estimator.

The two types of volume tensor estimation methods were used on the same set of neurons from a $40 \mu \mathrm{m}$ thick section from layer III of the medial frontal gyrus (MFG) of Brodmann Area 46 (BA46) in the human cerebral cortex (??). One formalin-fixed brain from a male patient with no history of neurological condition was selected from the brain collection at Core Centre for Molecular Morphology, Section for Stereology and Microscopy, Aarhus University Hospital, Aarhus, Denmark. The brain was collected in accordance with Danish law and with permission from the local ethical committee, see case no. 1-10-72-91-17. Data were obtained, using an Olympus BX51 light microscope with Olympus DP70 camera, an Olympus 60x oil lens (NA=1.35), prior motorized stage and newCAST software (Visiopharm, Hørsholm, Denmark).

The sampling of tissue is illustrated in Figure ??. Initially, BA46 was identified at the macroscopic level and the vertical axis was defined perpendicular to the pial surface. The tissue block was rotated uniformly around the vertical axis and placed in a container with $7 \%$ agar. After the agar was hardened, the block was cut into $2.5 \mathrm{~mm}$ thick parallel vertical slabs. Each slab was subsequently embedded in glycolmethacrylate (Technovit 7100) and cut into $40 \mu \mathrm{m}$ sections, stained with a Toluidinblue-Borax Solution $(1.33 \mathrm{~mL}$ Toluidinblue-Borax in $98.67 \mathrm{~mL}$ distilled water) for $30 \mathrm{~min}$, submerged in distilled water for $2 \mathrm{~min}$, air dired, mounted with Eukitt and covered with $120 \mu \mathrm{m}$ thick cover slips. The sample area was taken from the central gyral part of BA46, marked as a yellow rectangle in Figure ?? and was analyzed with a systematic set of disectors, resulting in 111 sampled neurons. The nucleolus of a neuron was used as reference point in the sampling. Figure ?? illustrates the collection of measurements for a sampled neuron, required for the section estimator. In the actual implementation, $n=4$ half lines were used. An illustration of the measurements required for the slice estimator may be found in ?, Fig. 7. Three optical planes were used for analysis of a sampled neuron and 
each optical plane was analyzed by two full lines. Since the expected number of intersection points is three times larger for the slice estimator than for the section estimator, the expected workload associated with the slice estimator is three times larger than that of the section estimator.

\section{- Figure ?? here -}

— Figure ?? here -

— Figure ?? here —

\section{Tensor data analysis}

In Table ??, we show the estimated mean particle volume $\widehat{v}$, the signed length of the estimated displacement vector $\widehat{c}$, the lengths of the semi-axes of the estimated Miles ellipsoid $\widehat{e}$ and the estimated elongation index, based on the section and the slice estimators, respectively. Recall that the elongation index is the ratio between the lengths of the semi-axes parallel and perpendicular to the vertical axis. Since we have taken a design-based approach and rotated the tissue block uniformly around the vertical axis, we do not need to assume rotational invariance and, in this case, $\widehat{c}$ estimates the average distance along the vertical axis from the nucleolus to the centre of mass of a neuron. Likewise, as explained earlier, the estimate of the elongation index is valid without rotational invariance. 
The estimated mean particle volumes, based on the section and the slice estimator, are quite similar and in fact equal to volumes of balls of radii $10.77 \mu \mathrm{m}$ and $10.99 \mathrm{\mu m}$, respectively. As a further investigation, we plot in Figure ?? the estimated particle volume, based on the slice estimator, against the estimated particle volume, based on the section estimator, for each of the 111 sampled neurons separately.

\section{— Figure ?? here -}

It is of course important to know the precision of the estimates, presented in Table ??. The variance of the volume estimators $\widehat{v}$ can be estimated by the empirical variance. The displacement vector is estimated by a ratio so, using a Taylor expansion of the ratio combined with empirical variances/covariances, we can obtain an estimate of the variance of the signed length of the displacement estimator. Estimation of the variances of the quantities relating to the Miles ellipsoid is more complicated. However, for any of the estimators, we can use classical resampling bootstrap to assess the variance, assuming that our sample is at least approximately independent and identically distributed (?). The results are given in Table ??. The alternative variance estimation methods mentioned above gave similar results, when applicable.

\section{— Table ?? here -}

Part of the variance of the section estimator is due to the random positioning of the half lines on the neuron profiles. For the slice estimator, the random positioning of the three optical planes and the lines within the optical planes contribute to the variance. In order to assess the magnitude of this design variance in relation to the total estimator variance, we performed 5 repeated measurements of 20 sampled neurons. Since the sampled neurons come from the same vertical slab, the remaining 
part of the total estimator variance includes variability due to the rotation of the slab around the vertical axis.

We will focus on estimation of mean particle volume, displacement and elongation index. The obtained estimates based on all data are shown in Table ??, together with the average time spent collecting a single set of measurements on one sampled neuron. Note that the estimated mean particle volumes, based on the section and the slice estimator, equal volumes of balls of radii $11.4 \mu \mathrm{m}$ and $11.7 \mu \mathrm{m}$, respectively. Note also that the time is approximately a factor 3 larger for the slice estimator than for the section estimator.

\section{— Table ?? here -}

In Tables ??-??, we assess the precision of the estimator of volume, displacement and elongation index in the case when a single set of measurements is available for 20 neurons. A bootstrap procedure can be used for this assessment, see Appendix B.

In Table ??, we show the components of the estimated variance for the mean particle volume estimator. The variance estimates have been determined empirically and by a bootstrap procedure, see Appendix B. When using bootstrap, the total variance may either be estimated as the sum of the average design variance and the particle variance (indicated by $(+)$ in Table ??) or by a separate procedure. Note that the obtained coefficient of variation $(\mathrm{CV})$ is $17 \%$ for both the section and the slice estimator. This is the precision of the estimated mean particle volume, when using a single set of measurements on 20 neurons.

\section{— Table ?? here -}

The components of the estimated variance of the displacement estimator, based on a single set of measurements on 20 neurons, may be found in Table ??. An estimate of the bias of the estimator of the signed length of the displacement may 
also be found in Table ??. As explained in Appendix B, the variance components may in the case of the section estimator be estimated, using a Taylor expansion or a bootstrap method. The estimated bias is small in absolute terms. Note that the displacement estimates obtained by the section and slice estimators in Table ?? are not significantly different, according to the estimated SDs in Table ??.

\section{— Table ?? here -}

In Table ??, the results for the elongation index shows a CV of $12 \%$ and $8 \%$ for the section and slice estimators, respectively. The estimated bias is again small in absolute terms.

\section{— Table ?? here -}

In Tables ??-??, the section and the slice estimator show similar performance, regarding bias and variance. However, the time spent for determining the slice estimator is a factor 3 longer than for the section estimator.

\section{Discussion}

In the present paper, we have described a new, simple stereological method of estimating volume tensors in 3D from vertical sections. In contrast to the earlier method, based on observation in several optical planes, see ?, the new method is less sensitive to tissue shrinkage in the direction of the z-axis, because for each sampled particle the section estimator only uses measurements in one optical plane, perpendicular to the $z$-axis. The section estimator is therefore not only useful for plastic sections but also for frozen or vibratome (agar embedding) sections, since these sections mainly shrink in the $z$-direction. Furthermore, in the examples considered in the present paper and in earlier simulation studies (?), the new estimator is more efficient than 
the one presented in ?. Also, the calculations needed for determining the section estimator are much simpler than those needed for the slice estimator.

Methods of assessing the bias and the precision of the new estimator, based on a bootstrap procedure, have also been provided in the present paper. Note that the estimator of mean particle volume is unbiased, while the estimators of displacement and elongation index may be biased to a degree, depending on the number of sampled particles. In the example, we found that the bias was small and in fact negligible if 100 neurons were sampled. The CVs obtained with 100 neurons were about $5 \%$ for volume and elongation while about $15 \%$ for displacement.

In a model-based setting, the new method requires that the particle population satisfies the assumption of rotational invariance with respect to the chosen vertical axis. A consequence of rotational invariance, that can be checked with the available observations, is the following. For each particle $X$, consider the profile $X \cap(x(X)+L)$, generated by the vertical plane centred at the reference point $x(X)$ of the particle. Reflect within $x(X)+L$ the profile in the vertical axis through $x(X)$. If rotational invariance is satisfied, the distribution of the reflected profiles will be the same as the distribution of the original profiles.

It is part of our future research plans to develop such procedures for checking rotational invariance. In the actual example from BA46, considered in the present paper, we adopted the design-based approach and used a vertical plane that was uniformly rotated around the vertical axis. This approach allowed us to estimate mean particle volume, the displacement in the direction of the vertical axis and the elongation index.

\section{Acknowledgements}

The brain studied in the present paper is part of a collection of brains, selected by Karl-Anton Dorph-Petersen and used in a larger brain study under publication. 
We thank Karl-Anton Dorph-Petersen for fruitful discussions concerning the choice of brain area. We sincerely thank Grazyna Rajkowska for assistance in delineating BA46. We are grateful to Kaj Vedel for the skilful assistance with the graphical illustrations. This work has been supported by Centre for Stochastic Geometry and Advanced Bioimaging, funded by the Villum Foundation, Sino-Danish Centre, Henny Sophie Clausen og MÃÿbelarkitekt Aksel Clausens Fond and Slagtermester Max WÃ̈̈rzner og Hustru Inger WÃ̈̈rzners Mindelegat.

\section{Appendix A - model-based approach}

The particle population of compact particles in $\mathbb{R}^{3}$ is modelled by a stationary marked point process

$$
\{[x(X) ; X-x(X)]\},
$$

where $x(X) \in X$ is a reference point of the particle $X$ and the mark $X-x(X)$ is the particle translated such that its reference point is at the origin $O$.

Let $\mathbf{X}_{0}$ be a random compact set distributed according to the particle mark distribution. The random set $\mathbf{X}_{0}$ may be considered as a typical particle with $O$ as its reference point. In this model-based approach, the mean particle volume tensor of rank $k$ is given by $\bar{T}_{k}=\mathbb{E} T_{k}\left(\mathbf{X}_{0}\right)$.

The estimator (??) is ratio-unbiased under this model-based approach. To see this, we use that for a function $f$ on compact subsets of $\mathbb{R}^{3}$

$$
\mathbb{E} \sum_{X \in S} f(X-x(X))=\mathbb{E} \sum_{x(X) \in W} f(X-x(X))=\lambda V(W) \mathbb{E} f\left(\mathbf{X}_{0}\right),
$$

where $\lambda=\mathbb{E} N(W) / V(W)$ is the intensity of the marked point process. It follows that

$$
\frac{\mathbb{E} \sum_{X \in S} f(X-x(X))}{\mathbb{E} N(W)}=\mathbb{E} f\left(\mathbf{X}_{0}\right)
$$


Choosing $f$ in (??) as the elements of $T_{k}$, we get

$$
\frac{\mathbb{E} \sum_{X \in S} T_{k}(X-x(X))}{\mathbb{E} N(W)}=\mathbb{E} T_{k}\left(\mathbf{X}_{0}\right),
$$

and the estimator (??) is therefore a ratio-unbiased estimator of $\bar{T}_{k}=\mathbb{E} T_{k}\left(\mathbf{X}_{0}\right)$.

The particle process is said to satisfy the rotational invariance assumption with respect to a line $M$ through $O$ if the distribution of $\mathbf{X}_{0}$ is invariant under rotations around $M$. The line $M$ is called the vertical axis, although $M$ may be an arbitrary line.

It follows from ?, (14.10) with $r=1,2$ that, under the rotational invariance assumption, the displacement vector $\bar{c}=\mathbb{E} T_{1}\left(\mathbf{X}_{0}\right) / \mathbb{E} T_{0}\left(\mathbf{X}_{0}\right)$ is parallel to the vertical axis and the Miles ellipsoid $\bar{e}$ is a centred ellipsoid of revolution around the vertical axis. In the particular case where the particle process is isotropic, $\bar{c}=O$ and $\bar{e}$ is a ball centred at $O$ with volume equal to the mean particle volume $\bar{v}$.

We will now derive the explicit form of $\widetilde{T}_{k}$ for $k=0,1,2$, along the lines in ?, p. 427-428. Using the definition of volume tensors, we find

$$
\begin{aligned}
\mathbb{E} T_{k}\left(\mathbf{X}_{0}\right) & =\frac{1}{k !} \mathbb{E} \int_{\mathbf{X}_{0}} x^{k} \mathrm{~d} x \\
& =\frac{1}{k !} \mathbb{E} \int_{\mathbb{R}^{3}} \mathbf{1}\left\{x \in \mathbf{X}_{0}\right\} x^{k} \mathrm{~d} x \\
& =\frac{1}{k !} \int_{\mathbb{R}^{3}} P\left\{x \in \mathbf{X}_{0}\right\} x^{k} \mathrm{~d} x
\end{aligned}
$$

where $\mathbf{1}\{\cdot\}$ is the notation used for the indicator function. Using cylindrical coordinates around the $z$-axis, we get

$$
\begin{aligned}
\mathbb{E} T_{k}\left(\mathbf{X}_{0}\right)=\frac{1}{k !} \int_{z=-\infty}^{\infty} \int_{u=0}^{\infty} \int_{\theta=0}^{2 \pi} & P\left((u \cos \theta, u \sin \theta, z) \in \mathbf{X}_{0}\right) \\
& \times(u \cos \theta, u \sin \theta, z)^{k} u \mathrm{~d} \theta \mathrm{d} u \mathrm{~d} z .
\end{aligned}
$$

Assuming rotational invariance with respect to the vertical axis, taken to be the 
$z$-axis, we find

$$
\mathbb{E} T_{k}\left(\mathbf{X}_{0}\right)=\int_{z=-\infty}^{\infty} \int_{u=0}^{\infty} P\left((u, 0, z) \in \mathbf{X}_{0}\right) f_{k}(u, z) \mathrm{d} u \mathrm{~d} z,
$$

where

$$
f_{k}(u, z)=\frac{1}{k !} \int_{\theta=0}^{2 \pi}(u \cos \theta, u \sin \theta, z)^{k} u \mathrm{~d} \theta
$$

and therefore,

$$
\mathbb{E} T_{k}\left(\mathbf{X}_{0}\right)=\mathbb{E} \int_{\mathbf{X}_{0} \cap L_{+}} f_{k}(u, z) \mathrm{d} u \mathrm{~d} z
$$

where $L_{+}$is the following half plane

$$
L_{+}=\{(u, 0, z): u>0, z \in \mathbb{R}\} .
$$

Using symmetry arguments, we finally get

$$
\mathbb{E} T_{k}\left(\mathbf{X}_{0}\right)=\frac{1}{2} \mathbb{E} \int_{\mathbf{X}_{0} \cap L} f_{k}(|u|, z) \mathrm{d} u \mathrm{~d} z
$$

where

$$
L=\{(u, 0, z): u, z \in \mathbb{R}\} .
$$

In conclusion,

$$
\check{T}_{k}\left(\mathbf{X}_{0} \cap L\right)=\frac{1}{2} \int_{\mathbf{X}_{0} \cap L} f_{k}(|u|, z) \mathrm{d} u \mathrm{~d} z
$$

is an unbiased estimator of $\mathbb{E} T_{k}\left(\mathbf{X}_{0}\right)$.

Using the formula for $f_{k}(u, z)$ given in ?, p. 428, we get for $k=0$

$$
\check{T}_{0}\left(\mathbf{X}_{0} \cap L\right)=\pi \int_{\mathbf{X}_{0} \cap L}|u| \mathrm{d} u \mathrm{~d} z
$$

while

$$
\check{T}_{1}\left(\mathbf{X}_{0} \cap L\right)=\left(0,0, \pi \int_{\mathbf{X}_{0} \cap L}|u| z \mathrm{~d} u \mathrm{~d} z\right)
$$


and $\check{T}_{2}\left(\mathbf{X}_{0} \cap L\right)$ is a $3 \times 3$ diagonal matrix with diagonal elements

$$
\check{T}_{2}\left(\mathbf{X}_{0} \cap L\right)_{11}=\check{T}_{2}\left(\mathbf{X}_{0} \cap L\right)_{22}=\frac{\pi}{4} \int_{\mathbf{X}_{0} \cap L}|u|^{3} \mathrm{~d} u \mathrm{~d} z
$$

and

$$
\check{T}_{2}\left(\mathbf{X}_{0} \cap L\right)_{33}=\frac{\pi}{2} \int_{\mathbf{X}_{0} \cap L}|u| z^{2} \mathrm{~d} u \mathrm{~d} z .
$$

Note that any of these integrals can be determined from information only within $\mathbf{X}_{0} \cap L$.

The estimators $\check{T}_{k}\left(\mathbf{X}_{0} \cap L\right)$ involve integrals of the form

$$
\int_{X \cap L}|u|^{i_{1}} z^{i_{2}} \mathrm{~d} u \mathrm{~d} z
$$

where $X$ is a compact subset of $\mathbb{R}^{3}$ and $i_{1}, i_{2}$ are non-negative integers. Note that $i_{1}$ is always an odd integer. If the profile $X \cap L$ is not available in digitized form, we may estimate the integral (??), using e.g. a line grid perpendicular to the $z$-axis. Let $L_{1}(z)$ denote the line in $L$, perpendicular to the $z$-axis, at height $z$. Let $z_{-}$and $z_{+}$be the lowest and highest point of the projection of $X \cap L$ onto the $z$-axis. Let $n$ be the number of lines, used in the line grid. The set of lines in the line grid is given by

$$
L_{1}\left(z_{i}\right), \quad i=1, \ldots, n
$$

where $z_{i}=U+\frac{i-1}{n}\left(z_{+}-z_{-}\right)$and $U \sim \operatorname{Unif}\left(z_{-}, z_{-}+\frac{z_{+}-z_{-}}{n}\right)$. Using the notation $t=$ $\left(z_{+}-z_{-}\right) / n$ from the main text, the integral (??) can then be estimated unbiasedly by

$$
t \sum_{i=1}^{n} \int_{X \cap L_{1}\left(z_{i}\right)}|u|^{i_{1}} \mathrm{~d} u \times z_{i}^{i_{2}} .
$$

Note that we may increase the efficiency of the estimation procedure by alternately choosing the positive and the negative half line with a random start, as shown in Figure ?? in the main text. In that case, the contribution from each half 
line should be multiplied by 2 in order to obtain an unbiased estimator of (??). Let us consider the contribution from a half line $L_{1+}\left(z_{i}\right)$. Define the power- $\alpha$ ray distance as

$$
l_{i}^{\alpha}=\sum_{j \text { even }} l_{i j}^{\alpha}-\sum_{j \text { odd }} l_{i j}^{\alpha}
$$

with $l_{i j}$ as introduced in the main text. Then,

$$
\int_{X \cap L_{1+}\left(z_{i}\right)}|u|^{i_{1}} \mathrm{~d} u \times z_{i}^{i_{2}}=\frac{1}{i_{1}+1} l_{i}^{i_{1}+1} z_{i}^{i_{2}}
$$

Using half lines, we get that

$$
\frac{2 t}{i_{1}+1} \sum_{i=1}^{n} l_{i}^{i_{1}+1} z_{i}^{i_{2}}
$$

is an unbiased estimator of (??). From this result, (??)-(??) follow.

As mentioned in the main text, rotational invariance may be introduced into the particle model by letting the vertical plane $L$ in (??) be distributed as $R L_{0}$, where $R$ is a uniform random rotation around $M$ and $L_{0}$ is a fixed vertical plane. The estimator $\widehat{T}_{k}$ can then be rewritten as

$$
\widehat{T}_{k}=\frac{1}{N(W)} \sum_{X \in S} \widetilde{T}_{k}\left(R^{-1}[X-x(X)] \cap L_{0}\right),
$$

where $R^{-1}$ is the inverse rotation. So an equivalent description of the situation is that each centred particle $X-x(X)$ is given a uniform random rotation and then sectioned by the fixed vertical plane $L_{0}$.

The induced particle model

$$
\left\{\left[x(X) ; R^{-1}(X-x(X))\right]\right\}
$$

satisfies the rotational invariance assumption with respect to $M$ with typical particle $\widetilde{\mathbf{X}}_{0}=R^{-1} \mathbf{X}_{0}$. Using that $R$ is a uniform random rotation around $M$, we get for any 
$u \in \mathbb{R}^{3}$

$$
\mathbb{E} R^{-1} u=P_{M} u,
$$

where $P_{M}$ is the orthogonal projection onto $M$, and it follows that

$$
\mathbb{E} T_{1}\left(\widetilde{\mathbf{X}}_{0}\right)=P_{M} \mathbb{E} T_{1}\left(\mathbf{X}_{0}\right) .
$$

Since $\mathbb{E} T_{0}\left(\widetilde{\mathbf{X}}_{0}\right)=\mathbb{E} T_{0}\left(\mathbf{X}_{0}\right)$, the displacement vector in the induced model is therefore equal to the projection of the displacement vector $\bar{c}$ in the original model onto

the vertical axis $M$. Accordingly, in the design-based approach, $\widehat{T}_{1} / \widehat{T}_{0}$ becomes an estimator of $P_{M} \bar{c}$.

As mentioned earlier, in the design-based approach we may imagine that each centred particle $X-x(X)$ is given a uniform random rotation around the vertical axis. Remaining shape information is available in the Miles ellipsoid of the induced model. Due to the fact that the induced model satisfies the rotational invariance assumption, this Miles ellipsoid is an ellipsoid of revolution around the vertical axis. The elongation index $I$ is the ratio between the lengths of the semi-axes of this ellipsoid, parallel and perpendicular to the vertical axis.

\section{Appendix B - bootstrap methods}

We have data of $r=5$ repeated measurements for each of $n=20$ neurons. The overall results (averaging over all 100 measurements) for the data are given in Table ??. We have also used the data to assess the precision of the proposed estimators in the case where a single set of measurements is available for each of $n=20$ particles. For this situation, Tables ??-?? summarize estimates of the different components of the variance for the volume estimator, the displacement and the elongation index using a bootstrap procedure (and a Taylor expansion approach where possible). For the volume estimator, we also estimated the variances by the empirical counterparts. 
The bootstrap procedures work as follows. Let $\mathbf{x}=\left(x_{k j}\right)_{k=1, \ldots, 20, j=1, \ldots, 5}$ be the collection of (vectorized) volume tensors estimated five times for each of $n=20$ particles from $r=5$ iid repeated measurements. Our aim is to assess the precision of the estimator of volume, displacement and elongation index in the case when $r=1$ measurement is available for $n=20$ particles. For a set $\mathbf{y}=\left(y_{k}\right)_{k=1, \ldots, 20}$ of one set of measurements per particle, we denote any of these estimators by $\theta(\mathbf{y})$. The overall estimate, averaging over all 100 measurements, is denoted by $\bar{\theta}$. The estimators we consider are not necessarily unbiased, so we aim to assess their bias, variance and coefficient of variation $(\mathrm{CV})$ by a bootstrap procedure. To do so, we draw $B=10^{5}$ bootstrap samples, where we first pick a sample $\left\{k_{1}, \ldots, k_{20}\right\}$ with replacement from the indices $1, \ldots, 20$ and then for each $k_{i}$, we pick a random index $j_{i} \in\{1, \ldots, 5\}$. Then, for each bootstrap sample $b=\left(\left(k_{i}, j_{i}\right)\right)_{i=1, \ldots, 20}$, we compute

$$
\theta_{b}^{*}=\theta\left(\left(x_{k_{i}, j_{i}}\right)_{i=1, \ldots, 20}\right)
$$

We obtain the following bootstrap estimates of bias and variance

$$
\begin{aligned}
\widehat{\operatorname{Bias}} & =\frac{1}{B} \sum_{b}\left(\theta_{b}^{*}-\bar{\theta}\right) \\
\widehat{\operatorname{Var}} & =\frac{1}{B-1} \sum_{b}\left(\theta_{b}^{*}-\frac{1}{B} \sum_{b} \theta_{b}^{*}\right)^{2}
\end{aligned}
$$

where $b$ denotes a bootstrap sample. This procedure was used to obtain the estimates of the total variance in the second last line in Tables ??-??.

The variance of $\theta$ can be decomposed as follows

$$
\operatorname{Var}(\theta(\mathbf{Y}))=\mathbb{E}(\operatorname{Var}(\theta(\mathbf{Y}) \mid P))+\operatorname{Var}(\mathbb{E}(\theta(\mathbf{Y}) \mid P))
$$

where $\mid P$ stands for "given the 20 particles" and $\mathbf{Y}$ is the random variable corresponding to the observation $\mathbf{y}$. We refer to the first part in the above decomposition as the average design variance and to the second part as the particle variance. 
For a bootstrap estimate of the average design variance, we proceed as follows. For $k=1, \ldots, 20$, we draw a bootstrap observation $j_{k} \in\{1, \ldots, 5\}$, and then we compute the variance as in (??) using

$$
\theta_{D, b}^{*}=\theta\left(\left(x_{k j_{k}}\right)_{k=1, \ldots, 20}\right)
$$

We also use a bootstrap procedure to estimate the particle variance. Here, we draw bootstrap samples $b=\left\{k_{1}, \ldots, k_{n}\right\}$ of size $n=20$ with replacement from $\{1, \ldots, 20\}$ and use $\theta_{P, b}^{*}$ to compute the variance as in (??), where

$$
\theta_{P, b}^{*}=\theta\left(\left(\frac{1}{5} \sum_{j=1}^{5} x_{k_{i} j}\right)_{i=1, \ldots, 20}\right)
$$

The bootstrap procedure yields two estimates of the total variance of $\theta(\mathbf{Y})$. One is obtained directly and one is the sum of the two bootstrap estimates of the average design variance and the particle variance. Both estimates agree well overall. The sum is marked with a $(+)$ in Tables ??-??.

For the volume estimators, we can alternatively estimate the total variance and the components of the variance in (??) by the empirical counterparts. For the section estimator used for the displacement, one can alternatively use a Taylor expansion approximation to estimate the variance components. We have

$$
\frac{T_{1}}{T_{0}} \approx \frac{\mathbb{E}\left(T_{1}\right)}{\mathbb{E}\left(T_{0}\right)}+\frac{1}{\mathbb{E}\left(T_{0}\right)}\left(T_{1}-\mathbb{E}\left(T_{1}\right)\right)-\frac{\mathbb{E}\left(T_{1}\right)}{\mathbb{E}\left(T_{0}\right)^{2}}\left(T_{0}-\mathbb{E}\left(T_{0}\right)\right)
$$


Hence

$$
\begin{aligned}
\operatorname{Var}\left(\frac{T_{1}}{T_{0}}\right) \approx & \frac{\operatorname{Var}\left(T_{1}\right)}{\mathbb{E}\left(T_{0}\right)^{2}}-2 \operatorname{Cov}\left(T_{1}, T_{0}\right) \frac{\mathbb{E}\left(T_{1}\right)}{\mathbb{E}\left(T_{0}\right)^{3}}+\operatorname{Var}\left(T_{0}\right) \frac{\mathbb{E}\left(T_{1}\right)^{2}}{\mathbb{E}\left(T_{0}\right)^{4}}, \\
\operatorname{Var}\left(\frac{T_{1}}{T_{0}} \mid P\right) \approx & \frac{\operatorname{Var}\left(T_{1} \mid P\right)}{\mathbb{E}\left(T_{0} \mid P\right)^{2}}-2 \operatorname{Cov}\left(T_{1}, T_{0} \mid P\right) \frac{\mathbb{E}\left(T_{1} \mid P\right)}{\mathbb{E}\left(T_{0} \mid P\right)^{3}} \\
& +\operatorname{Var}\left(T_{0} \mid P\right) \frac{\mathbb{E}\left(T_{1} \mid P\right)^{2}}{\mathbb{E}\left(T_{0} \mid P\right)^{4}}, \\
\operatorname{Var}\left(\mathbb{E}\left(\frac{T_{1}}{T_{0}} \mid P\right)\right) \approx & \frac{\operatorname{Var}\left(\mathbb{E}\left(T_{1} \mid P\right)\right)}{\mathbb{E}\left(T_{0}\right)^{2}}-2 \operatorname{Cov}\left(\mathbb{E}\left(T_{1} \mid P\right), \mathbb{E}\left(T_{0} \mid P\right)\right) \frac{\mathbb{E}\left(T_{1}\right)}{\mathbb{E}\left(T_{0}\right)^{3}} \\
& +\operatorname{Var}\left(\mathbb{E}\left(T_{0} \mid P\right)\right) \frac{\mathbb{E}\left(T_{1}\right)^{2}}{\mathbb{E}\left(T_{0}\right)^{4}}
\end{aligned}
$$

where our data allows us to estimate the quantities on the right hand side of the second and third line by their empirical counterparts. We estimate the total variance as the sum of the average design variance and the particle variance. 


\section{Figures and Tables moved here}

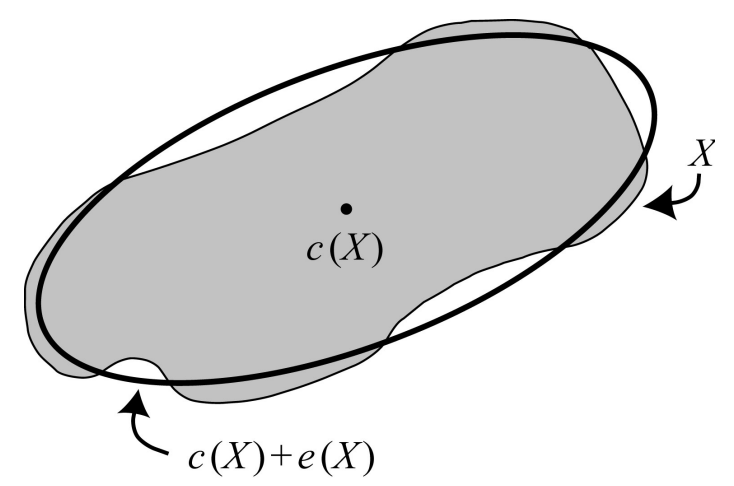

Figure 1: 2D illustration of the ellipsoidal approximation to a particle $X$ (grey). Here, $c(X)$ is the centre of mass of $X$ and $e(X)$ is a centred ellipsoid, approximating $X-c(X)$. If $X$ is an ellipsoid, $X=c(X)+e(X)$. 


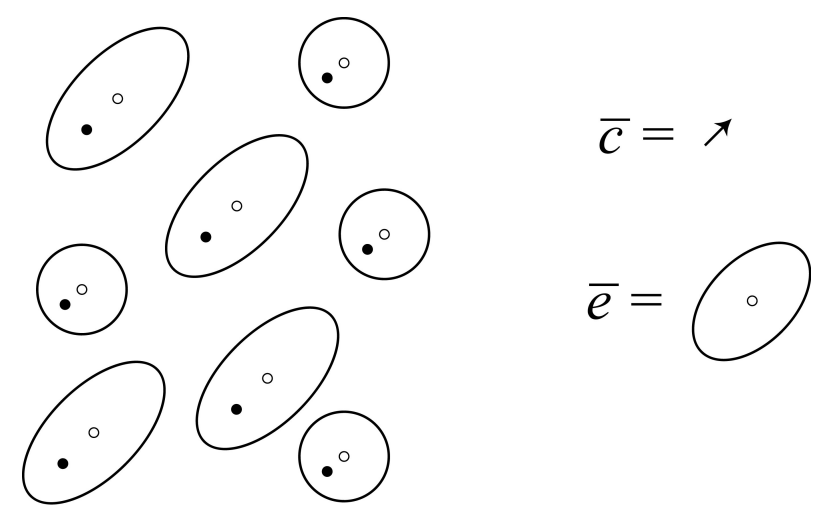

Figure 2: 2D illustration of the displacement vector $\bar{c}$ and the Miles ellipsoid $\bar{e}$ for a particle population, consisting of an equal mixture of ellipses and circular disks. The centre of mass of a particle is indicated by an open circle and the reference point by a closed circle. 


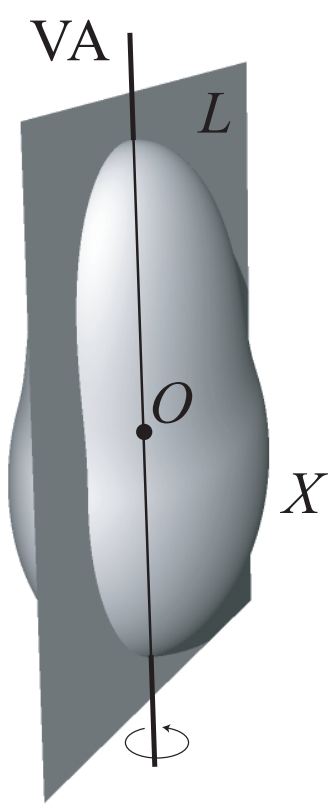

Figure 3: The particle $X$ is sectioned by a vertical plane $L$, containing the vertical axis (VA) and passing through the reference point $O$ of the particle. 


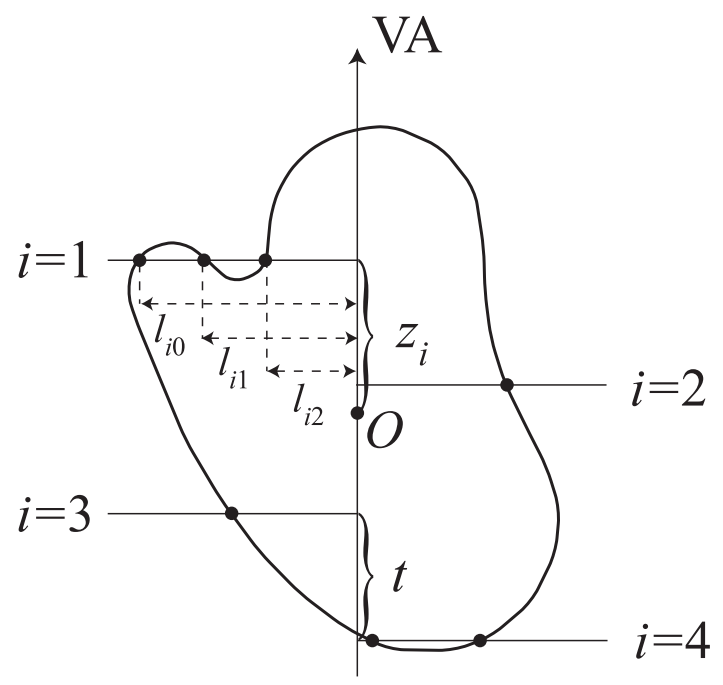

Figure 4: Illustration of the measurements on a sampled profile. A systematic set of alternating half lines, perpendicular to the vertical axis (VA), is used. The intersection points on a given half line are numbered according to decreasing distance to VA, using number 0 for the most distant intersection point. We let $l_{i j}$ be the distance from the $j$ 'th intersection point on the $i$ 'th half line to VA and $z_{i}$ is the (signed) distance from $O$ to the $i$ 'th half line. For more details, see the text. 


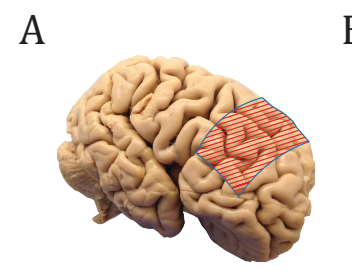

B

C

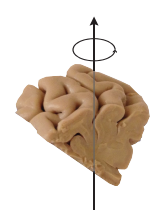

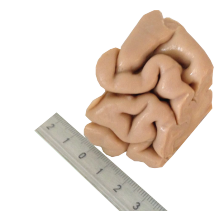

D

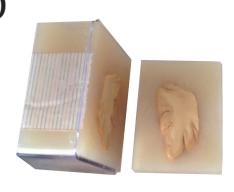

Figure 5: (A) The region BA46 is defined by its cytoarchitecture and is part of the Dorsolateral Prefrontal Cortex (DLPFC) which can be identified at the macroscopic level. (B) A coronal block of DLPFC. (C) The tissue block is rotated uniformly around a vertical axis (VA), perpendicular to the central pial surface of the block. (D) After agar hardening, the block is cut into $2.5 \mathrm{~mm}$ thick parallel vertical slabs. The slabs are embedded in glycolmethacrylate (Technovit 7100) and subsequently cut into $40 \mu \mathrm{m}$ thick sections that are stained with a Toluidinblue-Borax solution before further analysis. 


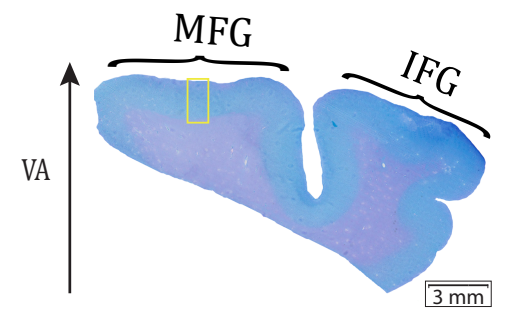

Figure 6: The sample area within the medial frontal gyrus (MFG) is marked with a yellow rectangle. The inferior frontal gyrus (IFG) is also indicated. VA is the vertical axis. 

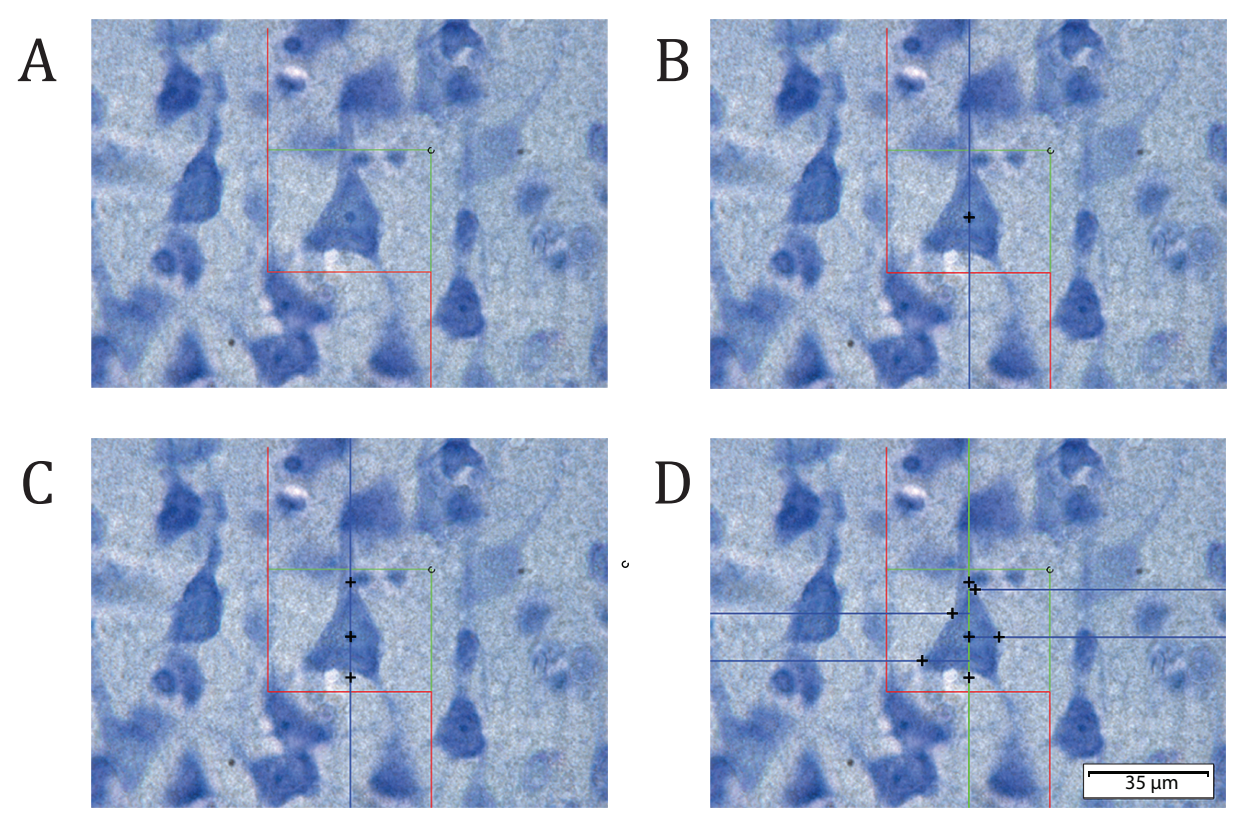

Figure 7: Measurement steps for a sampled neuron in layer III of the medial frontal gyrus in BA46, required for the section estimator. (A) A neuron in focus inside the optical disector. (B) The nucleolus was chosen as the reference point and the pre-defined vertical axis appears as a blue line. (C) The cell boundary in the vertical direction (top and bottom) are marked. (D) Four half lines perpendicular to the vertical axis with uniform random position appear. The intersection points between the neuron boundary and the half lines are marked with + . 


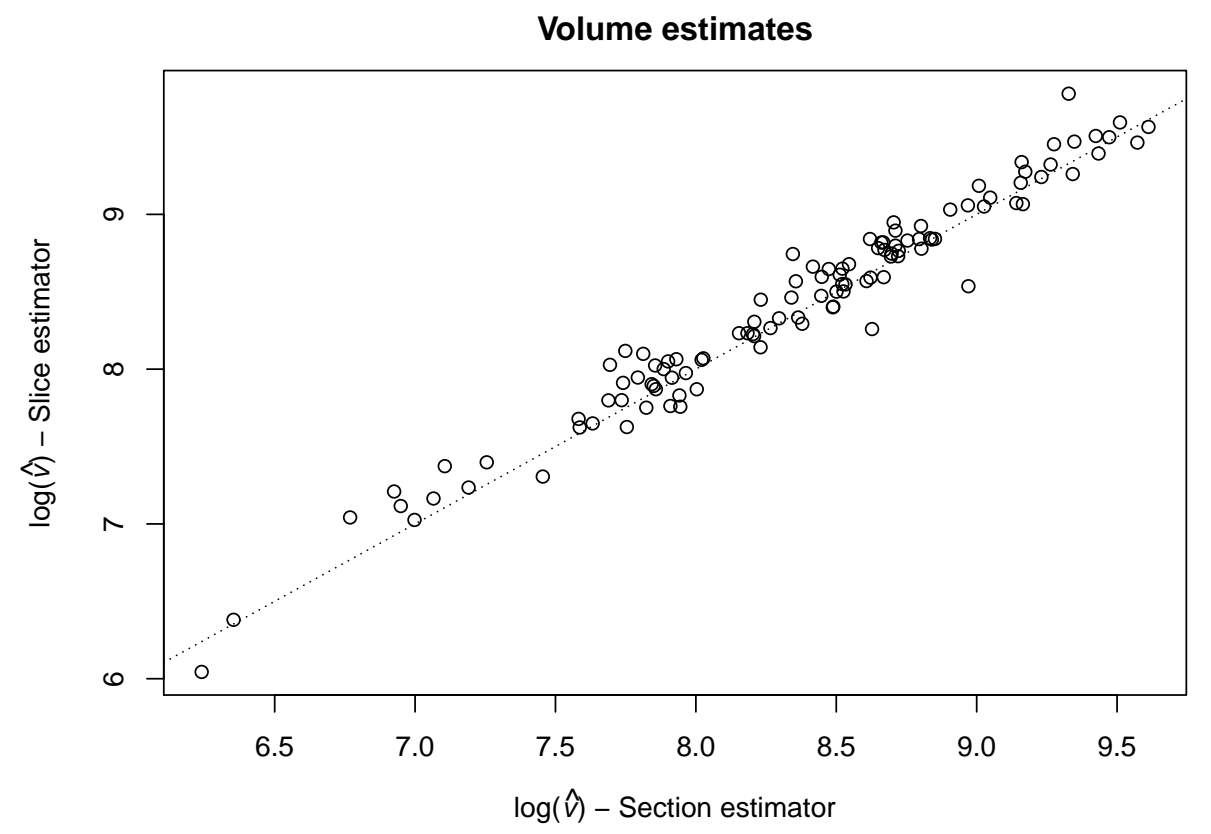

Figure 8: For each of the 111 sampled neurons, the volume estimate, based on the slice estimator, is plotted against the volume estimate, based on the section estimator. The dotted line is the identity. 
Table 1: The table illustrates for a sampled particle $X$ the measurements and calculations on the profile that enter into the estimates of mean particle volume tensors of rank 0,1 and 2. The measurements are in arbitrary units and refer to the profile shown in Figure ??. The distance between neighbour half planes is $t=1.2$. Using (??)-(??), we have $\widetilde{T}_{0}(X \cap L)=30.50$ and $\widetilde{T}_{1}(X \cap L)=(0,0,1.39)$, while $\widetilde{T}_{2}(X \cap L)$ is a diagonal matrix with first and second diagonal element equal to 11.43 and third diagonal element equal to 27.95 .

\begin{tabular}{ccccccccc}
\hline$i$ & $l_{i 0}$ & $l_{i 1}$ & $l_{i 2}$ & $z_{i}$ & $l_{i}^{2}$ & $z_{i} l_{i}^{2}$ & $z_{i}^{2} l_{i}^{2}$ & $l_{i}^{4}$ \\
\hline & & & & & & & & \\
1 & 2.1 & 1.4 & 0.9 & 1.4 & 3.26 & 4.564 & 6.3896 & 16.2626 \\
2 & 1.4 & & & 0.2 & 1.96 & 0.392 & 0.0784 & 3.8416 \\
3 & 1.2 & & & -1.0 & 1.44 & -1.440 & 1.4400 & 2.0736 \\
4 & 1.2 & 0.1 & & -2.2 & 1.43 & -3.146 & 6.9212 & 2.0735 \\
\hline & & & & & & & & \\
$\Sigma$ & & & & & 8.09 & 0.370 & 14.8292 & 24.2513 \\
\hline
\end{tabular}


Table 2: For the sample of 111 neurons, the table shows the estimated mean particle volume, the signed length of the estimated displacement vector, the lengths of the semiaxes of the estimated Miles ellipsoid and the estimated elongation index, based on the section and the slice estimators, respectively. For more details, see the text.

\begin{tabular}{lcc}
\hline & Section estimator & Slice estimator \\
\hline Volume $\left(\mu^{3}\right)$ & 5235 & 5558 \\
Displacement $(\mu \mathrm{m})$ & 1.86 & 2.00 \\
Parallel semi-axis $(\mu \mathrm{m})$ & 12.77 & 13.85 \\
Perpendicular semi-axes $(\mu \mathrm{m})$ & 9.89 & 9.79 \\
Elongation index & 1.29 & 1.41 \\
\hline
\end{tabular}


Table 3: Estimated bias and coefficient of variation for the sample of 111 neurons. Since the displacement vector may have zero length, the standard deviation is reported instead of the coefficient of variation for this parameter. For more details, see the text.

\begin{tabular}{llcc}
\hline & & Section estimator & Slice estimator \\
\cline { 3 - 4 } & & Bootstrap & Bootstrap \\
\hline Volume & CV & 0.059 & 0.060 \\
\hline Displacement & Bias & -0.003 & -0.001 \\
& SD & 0.309 & 0.289 \\
\hline Parallel & Bias & 0.004 & 0.002 \\
semi-axis & CV & 0.027 & 0.039 \\
\hline Perpendicular & Bias & -0.006 & -0.002 \\
semi-axes & CV & 0.026 & 0.027 \\
\hline Elongation & Bias & 0.002 & 0.002 \\
index & CV & 0.035 & 0.051 \\
\hline
\end{tabular}


Table 4: The table shows for the section and the slice estimators, respectively, the estimated mean particle volume, the signed length of the estimated displacement vector and the estimated elongation index, based on 5 repeated measurements of 20 sampled neurons. The average time spent collecting a single set of measurements on one sampled neuron is also shown.

\begin{tabular}{lcc}
\hline & Section estimator & Slice estimator \\
\hline Volume $\left(\mu^{3}\right)$ & 6151 & 6747 \\
Displacement $(\mu \mathrm{m})$ & 0.41 & 0.078 \\
Elongation index & 1.198 & 1.257 \\
\hline Time $(\mathrm{s})$ & 7.0 & 19.5 \\
\hline
\end{tabular}


Table 5: Components of the variance for volume estimators. For more details, see the text.

\begin{tabular}{lrrrrrr}
\hline & \multicolumn{2}{c}{ Section estimator } & & \multicolumn{2}{c}{ Slice estimator } \\
\cline { 2 - 3 } \cline { 5 - 6 } & Empirical & Bootstrap & & Empirical & Bootstrap \\
\hline Average design variance & 12836 & 10291 & & 76125 & 60149 \\
Particle variance & 1056755 & 1005402 & & 1226403 & 1165501 \\
Total variance & 1069590 & $(+) 1015693$ & & 1302528 & $(+) 1225650$ \\
& & 1027098 & & & 1230964 \\
\hline Total CV & 0.168 & 0.165 & & 0.169 & 0.164 \\
\hline
\end{tabular}


Table 6: Bias, variance and SD for the signed length of the displacement. For more details, see the text.

\begin{tabular}{lrrrr}
\hline & \multicolumn{2}{c}{ Section estimator } & & Slice estimator \\
\cline { 2 - 3 } \cline { 5 - 5 } & Taylor & Bootstrap & & Bootstrap \\
\hline Bias & - & 0.047 & & 0.017 \\
\hline Average design variance & 0.050 & 0.093 & & 0.100 \\
Particle variance & 0.941 & 0.877 & & 0.719 \\
Total variance & 0.991 & $(+) 0.970$ & & $(+) 0.820$ \\
& - & 0.967 & & 0.817 \\
\hline Total SD & 0.995 & 0.983 & & 0.904 \\
\hline
\end{tabular}


Table 7: Bias, variance and CV for the elongation index. For more details, see the text.

\begin{tabular}{lrrr}
\hline & Section estimator & & Slice estimator \\
\cline { 2 - 2 } Bias & Bootstrap & & Bootstrap \\
\hline Average design variance & 0.0260 & & 0.0044 \\
Particle variance & 0.0037 & & 0.0035 \\
Total variance & 0.0161 & & 0.0060 \\
& $(+) 0.0198$ & & $(+) 0.0095$ \\
Total CV & 0.0193 & & 0.0095 \\
\hline
\end{tabular}

